\title{
Construction and Performance Analysis of Variable-Weight Optical Orthogonal Codes for Asynchronous Optical CDMA Systems
}

\author{
Fong-Ray Gu and Jingshown Wu, Senior Member, IEEE
}

\begin{abstract}
In this paper, two construction schemes of variable-weight optical orthogonal codes (OOCs) for asynchronous optical-code-division multiple-access (O-CDMA) systems are proposed. The first scheme uses pairwise balanced design (PBD), which is a research topic in combinatorial theory. PBD produces a family of blocks with unequal block size. Therefore, PBD can be used to construct OOCs with variable code weight. The lower bound of the code size of the codes from PBD is formulated in this paper. A second scheme employes packing design with a partition to generate blocks with unequal block sizes. The variable-weight OOCs can be constructed by partitioning a larger weight codeword into a family of codes with a smaller code weight. The upper bound and lower bound of the code size of the second scheme are discussed. The bit-error-rate (BER) performances of the two proposed codes are evaluated analytically in this paper. The simulation results show that the codes from the first scheme have the same BER performance as that of conventional code, while the second scheme has a larger maximum number of simultaneous users than that of conventional codes.
\end{abstract}

Index Terms-Bit-error rate (BER), optical-code-division multiple access (O-CDMA), optical orthogonal codes (OOCs), packing design, pairwise balanced design (PBD), variable weight.

\section{INTRODUCTION}

$\mathbf{O}$ PTICAL-CODE-DIVISION multiple access (O-CDMA) is getting a lot of attention recently. It is a suitable technique for optical fiber transmission due to the inherent large bandwidth of fiber. It is also a good candidate for optical access networks, such as Ethernet passive optical networks (EPONs) [1], [2]. In the transmitter, a laser diode and an ON-OFF -keying (OOK) modulator are employed. Because of nonnegative power for optical signals, optical orthogonal codes (OOCs) are a family of $(0,1)$ sequences that are different from that in electrical transmission using $(+1,-1)$ sequences. The $\left(n, k, \lambda_{a}, \lambda_{c}\right)$-OOCs are a code family with code length $n$ and code weight $k$ [3]. The off-peak autocorrelation $\lambda_{a}$ and cross correlation $\lambda_{c}$ should be minimized for the sake of synchronization and less multipleuser interference (MUI). Meanwhile, the code weight $k$ is required to be large in order to distinguish the desired signal from MUI and noise. If $\lambda_{a}=\lambda_{c}=\lambda$, the $\left(n, k, \lambda_{a}, \lambda_{c}\right)$-OOCs are simply denoted as $(n, k, \lambda)$-OOCs. The $(n, k, \lambda)$-OOCs can

Manuscript received September 3, 2003; revised August 20, 2004. Part of this work was supported by the National Science Council and Ministry of Education, Taiwan, R.O.C., under Grants NSC91-2213-E-002-106 and 89-E-FA06-2-4.

The authors are with the Department of Electrical Engineering and Graduate Institute of Communication Engineering, National Taiwan University, Taipei 10617, Taiwan, R.O.C.

Digital Object Identifier 10.1109/JLT.2004.838880 be constructed from the projective geometry $P G(d, q)$, where $d$ is a positive integer $(d \geq 2)$ and $q$ is a prime power. The $P G(d, q)$ forms a $(n, v, \lambda)$ cyclic difference set with parameters $n=\left(q^{d+1}-1\right) /(q-1), v=\left(q^{d}-1\right) /(q-1)$, and $\lambda=\left(q^{d-1}-1\right) /(q-1)[4]$. It is also called the Singer difference set with classical parameters [5]. Any pair of the elements $\left(i, i^{\prime}\right)$ in the $v$ set of integers modulo $n$ has exactly $\lambda$ representations of the difference $i-i^{\prime}=\delta(\bmod n)$ for any residue $\delta \neq 0(\bmod n)$. Packing the $v$ elements of $P G(d, q)$ into $k$ subsets of blocks yields that every set of $(\lambda+1)$ distinct elements in $P G(d, q)$ occurs in at most one block. Then, a family of blocks can be obtained, and each block has $k$ elements. Each element in a block represents the mark position of the codeword, and therefore a family of OOCs can be generated. The $(\lambda+1)-(v, k, 1)$ packing design guarantees that the cross correlation of any two OOCs is $\lambda$. On the other hand, the property of the $(n, v, \lambda)$ cyclic difference set makes the off-peak autocorrelation of the OOCs to be $\lambda$ and therefore satisfies the correlation constraint of the $(n, k, \lambda)$-OOC's.

Let $\Phi(v, k, \lambda+1)$ be the possible number of blocks of a $(\lambda+$ $1)-(v, k, 1)$ packing design. Then, the upper bound is given as Theorem 1 [6].

Theorem 1 (Schönheim Bound):

$\Phi(v, k, \lambda+1) \leq\left\lfloor\frac{v}{k}\left\lfloor\frac{v-1}{k-1}\left\lfloor\frac{v-2}{k-2}\left\lfloor\cdots\left\lfloor\frac{v-\lambda}{k-\lambda}\right\rfloor \ldots\right\rfloor\right\rfloor\right\rfloor\right\rfloor$

where $\lfloor z\rfloor$ represents the integer part of $z$.

Theorem 1 can be followed to obtain the upper bound of code size of the $(n, k, \lambda)$-OOCs. In the literature, one way to improve the bit-error-rate (BER) performance of OOCs is to increase the code weight $k$ and decrease the cross correlation $\lambda$ [7]. In general, large code weight and small cross correlation will reduce the code size. Therefore, there is a tradeoff between the cross correlation and the code size of OOCs. In [8], Yang presented the construction of OOCs with unequal cross correlation. Recently, a family of $(n, k, 1,2)$-OOCs based on $(n, v, 1)$ perfect difference set is proposed [9]. The $(n, k, 1,2)$-OOCs also have better performance than $(n, k, 1,2)$ Yang's code and thus allow more simultaneous users under a given BER performance $\left(\leq 10^{-9}\right)$ [9]. A large code size can be obtained by relaxing the cross-correlation constraint of the OOCs. For example, the code size of $(n, k, 1,2)$-OOCs is about ten times larger than that of conventional $(n, k, 1)$-OOCs for reasonable code length. However, the BER performance degrades as the cross correlation increases. A recent paper discusses the performance bound of 
OOCs with large cross correlation [10]. For a given code length and the number of simultaneous users, the BER performance of the OOCs has a minimal value when the code weight is optimal.

Since the code size of OOCs depends upon the code weight, the variable-weight OOCs can generete larger code size than that of constant-weight OOCs. Meanwhile, subscribers with different code weights will have different BER performances. This property can meet the requirement of multimedia networks, which support multiple quality of services (QoS) [11]. Therefore, codewords of low code weight can be assigned to the low-QoS applications and high-code-weight codewords can be assigned to high-BER-requirement applications. In this paper, two construction schemes of variable-weight OOCs are proposed. The first scheme uses pairwise balanced design (PBD) to obtain a set of codewords with unequal code weight, whereas the second scheme employs packing design to partition a constant-weight codeword into subcodes with a smaller code weight. The performances of the codes produced by the two schemes are also analyzed.

This paper is organized as follows. Two construction schemes for the variable-weight OOCs are investigated in Section II. In Section III, the performance of the variable-weight OOCs is formulated using double optical hard limiters with consideration of thermal noise and shot noise. Numerical results are presented in Section IV. Finally, the conclusion is given in Section V.

\section{Construction Schemes of VARIABLE-Weight OOCs}

The variable-weight OOCs are a family of codewords with equal code length and variable code weight. The proposed schemes can be thought as a $(\lambda+1)-(v, K, 1)$ packing design with $r$ sets of blocks $K=\left\{k_{1}, k_{2}, \cdots, k_{r}\right\}$, where the block length $k_{i} \leq v$ for any $k_{i} \in K$. Based on the theory of combinatorial design, there exists a PBD that satisfies the requirement of the $(\lambda+1)-(v, K, 1)$ packing design. On the other hand, we can use the packing design twice to construct variable-weight OOCs under the fixed code length. We investigate two construction schemes for variable-weight OOCs as described in the following subsections.

\section{A. The Construction Scheme Using Pairwise Balanced Design}

A PBD of order $v$ is a pair $(X, \beta)$, where $X$ is a set of $v$ elements, and $\beta$ is a family of subsets (blocks) of $X$ with block sizes from $K=\left\{k_{1}, k_{2}, \cdots, k_{r}\right\}$. In a $(v, K, \lambda)$-PBD, every pair of distinct elements of $X$ occurs in exactly $\lambda$ blocks in $\beta$. When $\lambda=1$, the $(v, K, \lambda)$-PBD is usually denoted as $(v, K)$-PBD, which forms a linear space [12]. Then the blocks are called lines. The balanced incomplete block design (BIBD) is a special case of $(v, K, \lambda)$-PBD when $K=\{k\}$ is a singleton [13]. Define $\alpha(K)=\operatorname{gcd}\{k-1: k \in K\}$ and $\beta(K)=\operatorname{gcd}\{k(k-1)$ : $k \in K\}$, where $\operatorname{gcd}\{\cdot\}$ represents the greatest common divisor. Then, we have Theorem 2 [4].

Theorem 2: The necessary conditions for the existence of a $(v, K)$-PBD are

$$
v-1=0(\bmod \alpha(K))
$$

and

$$
v(v-1)=0(\bmod \beta(K)) .
$$

In the literature of combinatorial design, several existence theorems of $(v, K)$-PBD have been proven for some specified sets of $K$ [4]. Here, we introduce some of them, which will be used in this paper.

Theorem 3 [4]: $\mathrm{A}(v,\{3,4\})-\mathrm{PBD}$ exists if, and only if, $v=$ $0,1(\bmod 3)$, except when $v=6$.

Theorem 4 [4]: $\mathrm{A}(v,\{3,5\})-\mathrm{PBD}$ exists if, and only if, $v=$ $1(\bmod 2)$.

Theorem 5 [4]: $\mathrm{A}(v,\{4,5\})-\mathrm{PBD}$ exists if, and only if, $v=$ $0,1(\bmod 4)$, except when $v \in\{8,9,12\}$.

Theorem 6 [4]: A $(v,\{5,9\})-\mathrm{PBD}$ exists if, and only if, $v=$ $1(\bmod 4)$, except when $v \in\{13,17,29,33\}$ and possibly when $v \in\{93113173\}$.

Theorem 7 [14]: Let $q$ be a prime power. For any $0 \leq t \leq$ $q+1$, there exists a $\left(q^{2}+q+1-t,\{q-1, q, q+1\}\right)$-PBD.

Example 1: We consider a $(91,10,1)$ The Singer difference is set with elements $\{0,1,3,9,27,49,56,61,77,81\}$. Since $10=1 \bmod (3)$, then the $(10,(\{3,4\}))$-PBD exists. Therefore, we have the $(10,(\{3,4\}))-$ PBD with blocks: $\{1,27,61\}$, $\{1,49,77\}, \quad\{1,56,81\}, \quad\{3,27,81\}, \quad\{3,49,61\}, \quad\{3,56,77\}$, $\{9,27,77\},\{9,49,81\},\{9,56,61\},\{0,1,3,9\},\{0,27,49,56\}$, $\{0,61,77,81\}$.

For a set of $v$ elements in a $(v, K)$-PBD, the maximum possible number of blocks is clearly $\left(\begin{array}{l}v \\ 2\end{array}\right)$, where $\left(\begin{array}{l}x \\ y\end{array}\right)$ is the number of combinations of $x$ elements taken $y$ at a time. Erdös $e t$ al. [15] have shown that there is an absolute constant $c$ such that the possible number of blocks $g$ satisfies the inequality

$$
c v^{\frac{3}{2}}<g \leq\left(\begin{array}{l}
v \\
2
\end{array}\right), \quad g \neq\left(\begin{array}{l}
v \\
2
\end{array}\right)-1,\left(\begin{array}{l}
v \\
2
\end{array}\right)-3 .
$$

However, the upper bound of $g$ given $v$ elements and $K=$ $\left\{k_{1}, k_{2}, \cdots, k_{r}\right\}$ is still an unsolved problem. Several papers have discussed the lower bound of $g$ of a $(v, K)$-PBD [15]-[17]. At the given necessary conditions, there exists a linear space whose line lengths are $\left\{k_{1}, k_{2}, \cdots, k_{r}\right\}$. We have the following theorem.

Theorem 8: Let $k_{L}$ be the length of the longest blocks in the linear space of $v$ elements. The number of blocks in a $(v, K)$-PBD for a given set of block length $K=$ $\left\{k_{1}, k_{2}, \cdots, k_{L}\right\}$ satisfies

$$
g \geq 1+\frac{\left(v-k_{L}\right)\left[k_{L}\left(k_{s}+k_{s+1}-2\right)-v-1\right]}{\left(k_{s}-1\right)\left(k_{s+1}-1\right)} .
$$

The inequality holds for any $k_{s} \in K$.

Proof: Let $\ell_{k_{i}}$ be the number of blocks with length $k_{i}$, where $k_{i} \in K$. We define one of the blocks with length $k_{L}$ to be the base block. Then, the number of blocks with length $k_{L}$ except the base block is $\ell_{k_{L}}$. The following equation holds by counting the total blocks [16]:

$$
\sum_{k_{i} \in K} \ell_{k_{i}}+1=g
$$


In $(v, K)$-PBD, any two blocks have one element in common, and every pair of elements will appear in only one block. The total number of pairs of elements is

$$
\begin{aligned}
\sum_{k_{i} \in K} k_{i}\left(k_{i}-1\right) \ell_{k_{i}} & =v(v-1)-k_{L}\left(k_{L}-1\right) \\
& =\left(v-k_{L}\right)\left(v+k_{L}-1\right) .
\end{aligned}
$$

Define $\ell_{k_{i}, j}$ to be the number of blocks of length $k_{i}$ that include the $j$ th element of the base block. We have the following relation:

$$
\sum_{k_{i} \in K}\left(k_{i}-1\right) \ell_{k_{i}, j}=v-k_{L}
$$

where $j=1,2,3, \ldots, k_{L}$. The summation of (7) over $j$ is

$$
\sum_{j} \sum_{k_{i} \in K}\left(k_{i}-1\right) \ell_{k_{i}, j}=k_{L}\left(v-k_{L}\right) .
$$

If we count the number of blocks of length $k_{i}$ that pass the base block, we obtain

$$
\ell_{k_{i}}=\ell_{k_{i}, 0}+\ell_{k_{i}, 1}+\ell_{k_{i}, 2}+\cdots+\ell_{k_{i}, k_{L}}
$$

where the term $\ell_{k_{i}, 0}$ is the number of blocks of length $k_{i}$ that are disjoint from the base block. Combining (8) and (9), we obtain

$$
\sum_{k_{i} \in K}\left(k_{i}-1\right) \ell_{k_{i}}=k_{L}\left(v-k_{L}\right)+A
$$

where $A$ is given as

$$
A=\sum_{k_{i} \in K}\left(k_{i}-1\right) \ell_{k_{i}, 0}
$$

For a specific $k_{s} \in K$, we can multiply (5), (6), and (10) by $\left(k_{s}-1\right)\left(k_{s+1}-1\right), 1$, and $-\left(k_{s}+k_{s+1}-1\right)$, respectively, and sum them up to eliminate $\ell_{k_{s}}$ and $\ell_{k_{s+1}}$ to obtain

$$
\begin{aligned}
B=(g-1)\left(k_{s}-1\right) & \left(k_{s+1}-1\right)+\left[\left(v-k_{L}\right)\left(v+k_{L}-1\right)\right] \\
- & {\left[k_{L}\left(v-k_{L}\right)+A\right]\left(k_{s}+k_{s+1}-1\right) }
\end{aligned}
$$

where

$$
B=\sum_{\substack{k_{i} \in K \\ k_{i} \neq k_{s}, k_{q s+1}}}\left(k_{i}-k_{s}\right)\left(k_{i}-k_{s+1}\right) \ell_{k_{i}} .
$$

Denote $D=\left(k_{s}-1\right)\left(k_{s+1}-1\right)$, and we can express $g$ as

$$
\begin{aligned}
& g=1+\frac{\left(v-k_{L}\right)\left[k_{L}\left(k_{s}+k_{s+1}-2\right)-v+1\right]}{D} \\
& +\frac{A\left(k_{s}+k_{s+1}-1\right)}{D}+\frac{B}{D} \text {. }
\end{aligned}
$$

Since $A$ and $B$ are nonnegative, we have the lower bound of $g$ by dropping the terms containing $A$ and $B$ of (14). The result is

$$
g \geq 1+\left(v-k_{L}\right) \frac{\left[k_{L}\left(k_{s}+k_{s+1}-2\right)-v+1\right]}{D} .
$$

In (15), the equality holds when $A=B=0$. From (11), $A=$ 0 occurs when $\ell_{k_{i}, 0}=0$ for any $k_{i} \in K$, i.e., all blocks intersect the base block. From (13), $B=0$ implies $\left(k_{i}-k_{s}\right)\left(k_{i}-k_{s+1}\right)=$ 0 for any $k_{i} \in K$, which represents that the number of classes of block size in the PBD is less than or equal to two.

For some applications of the O-CDMA systems, we have only two classes of code weight $K=\left\{k_{1}, k_{2}\right\}$. Since there are only two variables, we can obtain the number of blocks by solving (6) and (10). For instance, the number of blocks with length 3 and 4 of a $(10,\{3,4\})-P B D$ can be obtained from

$$
\left\{\begin{array}{l}
6 \ell_{3}+12 \ell_{4}=(10-4)(10+4-1) \\
2 \ell_{3}+3 \ell_{4}=4(10-4)+A
\end{array} .\right.
$$

If we assume $A$ to be zero, i.e., all blocks in the (10, \{3,4\})-PBD intersect the base block, the possible number of blocks $g$ has a lower bound of 12 .

When the block length of a $(v, K)$-PBD is a set of successive integers $K=\left\{k_{1}, k_{1}+1, \cdots, k_{1}+r-1\right\}$, then (15) becomes

$$
g \geq 1+\frac{\left(v-k_{L}\right)\left[k_{L}\left(2 k_{s}-1\right)-v+1\right]}{k_{s}\left(k_{s}-1\right)} .
$$

We denote $F\left(k_{s}\right)$ as the right-hand side of (17) [17]. Then, we have

$$
F\left(k_{s}+1\right)-F\left(k_{s}\right)=\frac{2\left(v-k_{L}\right)\left(v-1-k_{s} k_{L}\right)}{\left(k_{s}-1\right) k_{s}\left(k_{s}+1\right)} .
$$

Equation (18) is nonnegative when $k_{s} \leq(v-1) / k_{L}$. Therefore, $F\left(k_{s}\right)$ is a monotonically increasing function when $k_{s} \leq(v-$ $1) / k_{L}$. Then, we can determine the strongest lower bound of $g$ by assigning $k_{s}=\left\lfloor(v-1) / k_{L}\right\rfloor[17]$.

The construction procedure of variable-weight OOCs by using PBD is described as the following.

1) Take an $(n, v, 1)$-Singer difference set with classical parameters $v=q^{a}+1$ and $n=q^{2 a}+q^{a}+1$, where $q$ is a prime power and $a$ is any positive integer. Any pair of the elements $\left(i, i^{\prime}\right)$ in the $(n, v, 1)$-Singer difference set has exactly one representation of the difference $i-i^{\prime}=\delta(\bmod n)$ for $i \neq i^{\prime}$.

2) Apply $(v, K)$-PBD on the $v$ elements of the $(n, v$, 1)-Singer difference set. A family of blocks with a block length from $K=\left\{k_{1}, k_{2}, \cdots, k_{L}\right\}$ can be obtained.

3) We can construct the variable-weight codes $C^{k_{i}}=$ $\left\{c_{0}^{k_{i}}, c_{1}^{k_{i}}, \cdots, c_{n-1}^{k_{i}}\right\}$ with code weight $k_{i}$ by mapping the elements of the PBD block into the code weight positions according to the following rule:

$$
c_{l}^{k_{i}}= \begin{cases}1, & \text { if } l \in(v, K)-\text { PBD block } \\ 0, & \text { otherwise }\end{cases}
$$

\section{B. The Construction Scheme Using Packing Design With Partition}

We next investigate the construction scheme of variable-weight OOCs using packing design with a partition of 
elements in the projective plane $P G(2, q)$. A standard result of the existence of $P G(2, q)$ is given in Theorem 9 .

Theorem 9 [14]: The $P G(2, q)$ is a BIBD with parameter $\left(q^{2 a}+q^{a}+1, q^{a}+1\right), 1$, where $q$ is a prime power and $a$ is a positive integer.

Theorem 10[4]: Let $a$ and $b$ be positive integers such that $b$ divides $a$. If there exists $P G\left(2, q^{a}\right)$, then it contains a subplane of $P G\left(2, q^{b}\right)$.

If $q$ is a prime power and $a$ is any positive integer, a finite Möbius geometry of $M G(q, a)$ is an extended field $F$, which includes an element $\infty$ into a Galois field $G F\left(q^{a}\right)$. The $M G(q, a)$ has $q^{a}+1$ elements. Any triple of elements in $M G(q, a)$ is included in exactly one block, and every block has $q^{b}+1$ elements, where $b$ is a positive integer that divides $a$. Meanwhile, an $(n, v, 1)$-Singer difference set with classical parameters of $n=q^{2 a}+q^{a}+1$ has $v=q^{a}+1$ elements. The $q^{a}+1$ elements form a plane of $P G\left(2, q^{a}\right)$. Therefore, we can put the $q^{a}+1 \mathrm{el}-$ ements of the Singer difference set into the plane of $M G(q, a)$ [9]. We have the following theorem.

Theorem 11: A finite Möbius geometry of $M G(q, a)$ contains a subplane of $M G(q, b)$ if $b$ is a positive integer that divides $a$.

Proof: According to Theorem 10, $P G\left(2, q^{a}\right)$ contains a subplane of $P G\left(2, q^{b}\right)$ when $b$ divides $a$. Since the elements in the $P G\left(2, q^{a}\right)$ form a plane of $M G(q, a)$, then the $M G(q, a)$ also contains a subplane of $M G(q, b)$.

If we put the elements of an $(n, v, 1)$-Singer difference set with classical parameters into the plane of $M G(q, a)$, we can use $3-\left(q^{a}+1, q^{b}+1,1\right)$ packing design to construct a family of blocks with block length of $q^{b}+1$. Then, the total number of blocks is given as

$$
\Phi\left(q^{a}+1, q^{b}+1,3\right)=\left\lfloor\frac{q^{a}+1}{q^{b}+1}\left\lfloor\frac{q^{a}}{q^{b}}\left\lfloor\frac{q^{a}-1}{q^{b}-1}\right\rfloor\right\rfloor\right\rfloor .
$$

Equation (20) is the upper bound of the packing design, and the $3-\left(q^{a}+1, q^{b}+1,1\right)$ packing design is called the maximal system [6]. In order to obtain a family of blocks with different block sizes, we propose a construction scheme that uses packing design twice. The $q^{a}+1$ elements in $M G(q, a)$ are first packed into blocks by a $3-\left(q^{a}+1, q^{b}+1,1\right)$ packing design. Next, a block with $q^{b}+1$ elements is partitioned into subblocks, and each subblock has length $k_{i}$ and $2 \leq k_{i} \leq q^{b}+1$. A family of subblocks with different block length is then constructed. Each entry in the subblock represents the code weight position of a codeword, and then the subblocks with variable length can construct a family of variable-weight codewords. The construction procedure of this proposed scheme is listed in the following.

1) Construct an $(n, v, 1)$-Singer difference set with classical parameters $v=q^{a}+1$ and $n=q^{2 a}+q^{a}+1$, where $q$ is a prime power and $a$ is any positive integer.

2) Pack the $q^{a}+1$ elements of the Singer difference set into blocks with length $q^{b}+1$ by $3-\left(q^{a}+1, q^{b}+1,1\right)$ packing design, where $b$ is a positive integer that divides $a$.

3) Wrap the $q^{b}+1$ elements of one block into smaller blocks with length $k_{i}$ such that any two subblocks have one element in common. Therefore, a family of subblocks with size $k_{i}$ is generated.
4) Each entry of a block represents the weight position of the codeword. We can obtain a variable code weight $\left(n,\left\{k_{1}, k_{2}, \cdots, k_{r}\right\}, 1,2\right)$-OOCs with $r$ classes of code weight.

According to the packing design, the number of subblocks with length $k_{i}$ from $q^{b}+1$ elements is upper bounded by

$$
\Phi\left(q^{b}+1, k_{i}, 2\right) \leq\left\lfloor\frac{q^{b}+1}{k_{i}}\left\lfloor\frac{q^{b}}{k_{i}-1}\right\rfloor\right\rfloor .
$$

The low bound of $\Phi\left(q^{b}+1, k_{i}, 2\right)$ can be considered as the number of subblocks by using the brute force method, that is, at least the number of subblocks we can construct. We prove the lower bound of $\Phi\left(q^{b}+1, k_{i}, 2\right)$ in the following.

Theorem 12: The lower bound of wrapping $\left(q^{b}+1\right)$ elements into smaller blocks with $k_{i}$ elements is given as

$$
\Phi\left(q^{b}+1, k_{i}, 2\right) \geq \chi+\left\lfloor\frac{\chi+\psi-1}{k_{i}-1}\right\rfloor \times\left(k_{i}-1\right)
$$

where $\chi=\left\lfloor\left(q^{b} / k_{i}-1\right)\right\rfloor$ and $\psi=q^{b}-\chi \cdot\left(k_{i}-1\right)$.

Proof: Among the $\left(q^{b}+1\right)$ elements, we choose one element as the intersecting point and then partition the rest of the $q^{b}$ elements into groups, and each group has $\left(k_{i}-1\right)$ elements. Then, the number of groups is $\chi$, and $\chi=\left\lfloor\left(q^{b} / k_{i}-1\right)\right\rfloor$. The residual elements after grouping is denoted as $\psi$, and $\psi=$ $q^{b}-\chi \cdot\left(k_{i}-1\right)$. By putting the intersecting point into every group, we can obtain a family of blocks with block size $k_{i}$. Any two blocks intersect only at the intersecting point, and there are $\chi$ blocks. In the next step, we use the same method to construct blocks from the $\chi$ groups and the $\psi$ residual elements. Since the groups without the intersecting point are disjoint with each other, we can pick one element from each group except the intersecting point to satisfy the correlation property. Then, the number of blocks obtained in this step is $\lfloor(\chi+\psi-$ 1) $\left./\left(k_{i}-1\right)\right\rfloor \times\left(k_{i}-1\right)$. Therefore, the total number of blocks is $\chi+\left\lfloor(\chi+\psi-1) /\left(k_{i}-1\right)\right\rfloor \times\left(k_{i}-1\right)$.

Theorem 13: The off-peak autocorrelation of the (n, $\left.\left\{k_{1}, k_{2}, \cdots k_{r}\right\}, 1,2\right)$-OOCs is less than or equal to one.

Proof: According to Step 1 of the construction procedure, the variable-weight OOCs are from the $(n, v, 1)$-Singer difference set. Then, any pair of the elements $\left(i, i^{\prime}\right)$ in the difference set has exactly one representation of the difference $i-i^{\prime}=$ $\delta(\bmod n)$ for any residue $\delta \neq 0(\bmod n)$. Therefore, after any time shift $\tau$, the off-peak autocorrelation of the codeword is less than or equal to one.

Theorem 14: The cross correlation of any two codewords originated from the same block in $M G(q, a)$ is one.

Proof: Any two codewords originated from the same block satisfy the correlation constraint of $2-\left(q^{b}+1, k_{i}, 1\right)$ packing design, in which any two subblocks have at most one element in common. Therefore, the cross correlation $\lambda_{c}$ of any two codewords originated from the same block is one.

Theorem 15: The cross correlation of any two codewords originated from different blocks in $M G(q, a)$ is less then or equal to two.

Proof: The family of subblocks originated from a block in $M G(q, a)$ by a $2-\left(q^{b}+1, k_{i}, 1\right)$ packing design. Because any two blocks in $M G(q, a)$ have two elements in common, then 


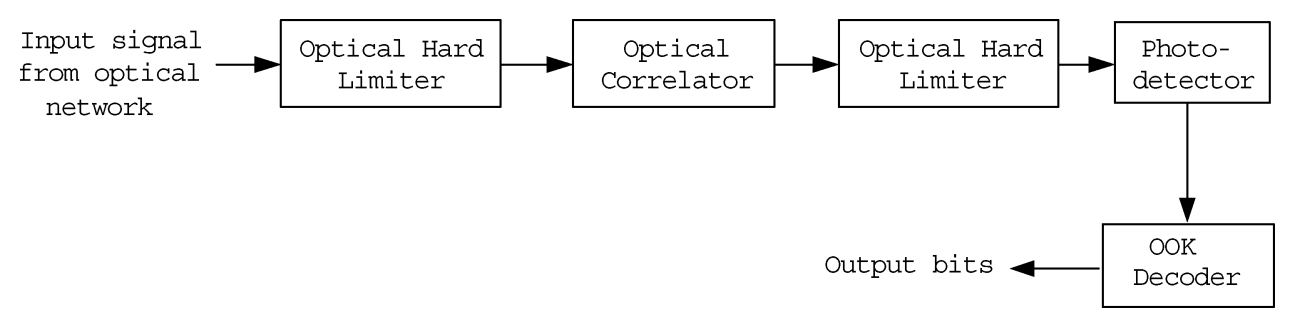

Fig. 1. Receiver structure of asynchronous O-CDMA systems using double optical hard limiters.

any two subblocks that originated from different blocks will intersect no more than two elements. Therefore the cross correlation of any two codewords originated from different blocks of $M G(q, a)$ will be less than or equal to two.

Let the fractions of the codewords with weight $\left\{k_{1}, k_{2}, \cdots, k_{r}\right\}$ be $\left\{p_{1}, p_{2}, \cdots, p_{r}\right\}$, respectively. The number of cardinalities of the variable-weight OOCs can be expressed as

$$
g=\frac{\Phi\left(q^{a}+1, q^{b}+1,3\right)}{E}
$$

where $E=\sum_{i=1}^{r} p_{i} / \Phi\left(q^{b}+1, k_{i}, 2\right)$.

\section{PERFormance ANALYSIS}

We analyze the performance of the asynchronous O-CDMA systems using the proposed variable-weight OOCs and double hard limiters with consideration of shot noise, thermal noise, avalanche photodiode (APD) bulk and surface leakage currents. The receiver structure is shown in Fig. 1 [18]. For convenience, we assume that the system is chip synchronous among users since it is the worst case of the performance [7].

The average photon arrival rate $\lambda$ per pulse is given by

$$
\lambda=\frac{\eta P_{W}}{h f}
$$

where $\eta$ is the APD quantum efficiency, $P_{W}$ is the received signal power, $h$ is the Planck's constant, and $f$ is the optical frequency. The optical signal power after the second hard limiter will be limited to two levels: ON and OFF levels. We denote state $S_{1}$ as the optical signal power of the ON level and state $S_{0}$ as the OFF level. The average photon arrival rate is $\lambda$ for state $S_{1}$; otherwise, the photon arrival rate is zero. For state $S_{i}, i \in\{0,1\}$, the probability density function (pdf) of the output current $Y_{i}$ of the photodetector is assumed Gaussian given by [19]

$$
P_{Y_{i}}\left(y_{i}\right)=\frac{1}{\sqrt{2 \pi \sigma_{i}^{2}}} e^{-\frac{\left(y_{i}-\mu_{i}\right)^{2}}{2 \sigma_{i}^{2}}}
$$

where $\mu_{i}$ is the mean value of the photodetector output current given by

$$
\mu_{i}=G T_{c}\left(i \lambda+\frac{I_{b}}{e}\right)+\frac{T_{c} I_{s}}{e}
$$

where $G$ is the average APD gain, $T_{c}$ is the chip time, $e$ is the electron charge, $I_{b} / e$ is the contribution of the APD bulk leakage current to the APD output, and $I_{s}$ is the APD surface leakage current. The variance of the photocurrent $\sigma_{i}^{2}$ can be expressed as

$$
\sigma_{i}^{2}=G^{2} F_{e} T_{c}\left(i \lambda+\frac{I_{b}}{e}\right)+\frac{T_{c} I_{s}}{e}+\sigma_{\mathrm{th}}^{2}
$$

where $F_{e}$ is the excess noise factor given by

$$
F_{e}=k_{\mathrm{eff}} G+\left(2-\frac{1}{G}\right)\left(1-k_{\mathrm{eff}}\right) .
$$

Here, $k_{\text {eff }}$ is the APD effective ionization ratio, and $\sigma_{\text {th }}^{2}$ is the variance of thermal noise expressed as

$$
\sigma_{\mathrm{th}}^{2}=\frac{2 k_{B} T_{r} T_{c}}{e^{2} R_{L}}
$$

where $k_{B}$ is the Boltzmann's constant, $T_{r}$ is the receiver noise temperature, and $R_{L}$ is the receiver load resistance.

In order to minimize the error probability, we set the threshold of the decision circuit $\theta$ as

$$
\theta=\frac{\mu_{0} \sigma_{1}+\mu_{1} \sigma_{0}}{\sigma_{1}+\sigma_{0}} .
$$

If the output current $Y_{i}$ is larger than $\theta$, the output data bit $b_{o}$ is decided to be bit one, or otherwise bit zero. The probability that the state error occurs is given by

$$
\begin{aligned}
& \operatorname{Pr}\left(b_{o}=1 \mid S_{0}\right)=\frac{1}{2} \operatorname{erfc}\left(\frac{\theta-\mu_{0}}{\sqrt{2 \sigma_{0}^{2}}}\right) \\
& \operatorname{Pr}\left(b_{o}=0 \mid S_{1}\right)=\frac{1}{2} \operatorname{erfc}\left(\frac{\mu_{1}-\theta}{\sqrt{2 \sigma_{1}^{2}}}\right)
\end{aligned}
$$

where $\operatorname{erfc}(\cdot)$ is the complementary error function and can be expressed as

$$
\operatorname{erfc}(z)=\frac{2}{\sqrt{\pi}} \int_{z}^{\infty} \exp \left(-u^{2}\right) d u
$$

Denote $U^{k_{i}}$ as the user using spreading code $C^{k_{i}}=$ $\left\{c_{0}^{k_{i}}, c_{1}^{k_{i}}, \cdots, c_{n-1}^{k_{i}}\right\}$ with Hamming weight $k_{i}$. If the relative frame offset between the desired user $U^{k_{i}}$ and the interfering user $U^{k_{j}}$ is $\tau$, then the cross correlation can be expressed as

$$
I_{\tau}=\sum_{l=0}^{n-1} c_{l}^{k_{i}} c_{l+\tau}^{k_{j}}
$$

The probability that one specified mark position of user $U^{k_{i}}$ hit by the interfering user $U^{k_{j}}$ is $k_{j} / 2 n$, where the factor $1 / 2$ 
TABLE I

Number of CARdinalities of THE Proposed VARIABle-Weight $\left(n,\left\{k_{1}, k_{2}\right\}\right)$-OOCs AND CONVENTIONAL $\left(n,\left\{k_{1}, k_{2}\right\}, 1,1,\{2 / 3,1 / 3\}\right)$-OOCs

\begin{tabular}{c|c|c|c|c}
\hline Code Type & $\begin{array}{c}\text { Convention } \\
\text { upper bound }\end{array}$ & $\begin{array}{c}\text { Scheme 1 } \\
\text { lower bound }\end{array}$ & $\begin{array}{c}\text { Scheme 2 } \\
\text { lower bound }\end{array}$ & $\begin{array}{c}\text { Scheme 2 } \\
\text { upper bound }\end{array}$ \\
\hline \hline$(91,\{4,3\})$ & 9 & 12 & 30 & 30 \\
\hline$(273,\{5,4\})$ & 15 & 18 & 67 & 67 \\
\hline$(273,\{5,3\})$ & 18 & 19 & 79 & 85 \\
\hline$(651,\{6,5\})$ & 24 & 28 & 129 & 129 \\
\hline$(651,\{6,3\})$ & 28 & 31 & 172 & 172 \\
\hline$(2451,\{8,7\})$ & 48 & 54 & 349 & 349 \\
\hline$(2451,\{8,6\})$ & 51 & 55 & 349 & 349 \\
\hline$(2451,\{8,4\})$ & 60 & 59 & 420 & 465 \\
\hline$(4161,\{9,8\})$ & 63 & 70 & 519 & 519 \\
\hline$(4161,\{9,6\})$ & 72 & 73 & 519 & 519 \\
\hline$(4161,\{9,5\})$ & 75 & 75 & 624 & 668 \\
\hline$(6643,\{10,9\})$ & 78 & 88 & 738 & 738 \\
\hline$(6643,\{10,8\})$ & 84 & 89 & 738 & 738 \\
\hline$(6643,\{10,5\})$ & 99 & 95 & 885 & 984 \\
\hline$(6643,\{10,4\})$ & 102 & 99 & 948 & 1032 \\
\hline
\end{tabular}

represents that each user transmits data 0 and 1 with equal probability. Since user $U^{k_{i}}$ has $k_{i}$ marks, then the expected value of $I_{\tau}$ interfered by user $U^{k_{j}}$ is given as

$$
E\left(I_{\tau}\right)=k_{i} \cdot \frac{k_{j}}{2 n} .
$$

For the first construction scheme, the probability of $I_{\tau}=2$ is zero because the codes are constructed from $(v, K)$-PBD, and the cross correlation of any two codewords is less than two. For the second construction scheme, the event $I_{\tau}=2$ occurs when the frame offset $\tau=0$ and the desired user $U^{k_{i}}$ and the interfering user $U^{k_{j}}$ originated from different blocks in $M G(q, a)$. Moreover, according to Step 3 of the construction procedure, a block in $M G(q, a)$ is partitioned into subblocks with smaller size. The probability that any two codewords with code weight $k_{i}$ and $k_{j}$, respectively, have two identical mark positions after the partition is $\xi$, and $\xi=\left(\left(\begin{array}{c}q^{b}-1 \\ k_{i}-2\end{array}\right) /\left(\begin{array}{c}q^{b}+1 \\ k_{i}\end{array}\right)\right)\left(\left(\begin{array}{c}q^{b}-1 \\ k_{j}-2\end{array}\right) /\left(\begin{array}{c}q^{b}+1 \\ k_{j}\end{array}\right)\right)$. Hence, the probability of $I_{\tau}=2$ is given by

$$
\begin{aligned}
\operatorname{Pr}\left(I_{\tau}\right. & =2) \\
& = \begin{cases}0, & \text { if } U^{k_{j}} \text { is from the same block as } U^{k_{i}} \\
\frac{1}{n} \cdot \xi, & \text { otherwise. }\end{cases}
\end{aligned}
$$

The probability that the interfering user $U^{k_{j}}$ contributes two interfering marks is denoted as $\gamma_{2}^{k_{j}}$ and given by

$$
\gamma_{2}^{k_{j}}=\frac{\operatorname{Pr}\left(I_{\tau}=2\right)}{2}
$$

where the factor $1 / 2$ represents that each user transmits data 0 and 1 with equal probability. Let $\gamma_{1}^{k_{j}}$ be the probability that user $U^{k_{j}}$ contributes one interfering mark. Then, the value of $\gamma_{1}^{k_{j}}$ can be obtained from (35) and (37) as follows:

$$
\gamma_{1}^{k_{j}}=E\left(I_{\tau}\right)-2 \cdot \gamma_{2}^{k_{j}} .
$$

We denote $F_{s}^{k_{j}}$ as the probability that any $s$ marks of the desired codeword is not interfered by $U^{k_{j}}$ and is given by

$$
F_{s}^{k_{j}}=1-\gamma_{1}^{k_{j}}-\gamma_{2}^{k_{j}}+\gamma_{1}^{k_{j}} \frac{k_{i}-s}{k_{i}}+\gamma_{2}^{k_{j}} \frac{\left(\begin{array}{c}
k_{i}-s \\
2
\end{array}\right)}{\left(\begin{array}{c}
k_{i} \\
2
\end{array}\right)} .
$$

For the receiver with double hard limiters, the signal state at the second hard-limiter output will be $S_{1}$ if the transmitted data bit $b$ is 1 , i.e., $\operatorname{Pr}\left(S_{1} \mid b=1\right)=1$. If the transmitted data bit is 0 , the second hard -limiter cannot entirely remove the MUI when the number of interfering marks exceeds or equals the code weight $k_{i}$. We assume that for the desired user $U^{k_{i}}$, there are $\left\{m_{1}, m_{2}, \cdots, m_{r}\right\}$ simultaneous users, where $m_{k}$ is the number of simultaneous users of code weight $k_{k}$, and $r$ is the number of classes of different weights. Then, the probability that an error occurs at the output state of the second hard limiter can be formulated as [11]

$$
\operatorname{Pr}\left(S_{1} \mid b=0\right)=\sum_{s=0}^{k_{i}}(-1)^{s}\left(\begin{array}{c}
k_{i} \\
s
\end{array}\right)\left(F_{s}^{k_{i}}\right)^{m_{i}-1} \prod_{\substack{j=1 \\
j \neq i}}^{r}\left(F_{s}^{k_{j}}\right)^{m_{j}} .
$$

The BER is given as

$$
\begin{aligned}
\mathrm{BER}= & \operatorname{Pr}\left(b_{o}=1 \mid S_{0}, b=0\right) \operatorname{Pr}\left(S_{0} \mid b=0\right) \operatorname{Pr}(b=0) \\
& +\operatorname{Pr}\left(b_{o}=1 \mid S_{1}, b=0\right) \operatorname{Pr}\left(S_{1} \mid b=0\right) \operatorname{Pr}(b=0) \\
& +\operatorname{Pr}\left(b_{o}=0 \mid S_{0}, b=1\right) \operatorname{Pr}\left(S_{0} \mid b=1\right) \operatorname{Pr}(b=1) \\
& +\operatorname{Pr}\left(b_{o}=0 \mid S_{1}, b=1\right) \operatorname{Pr}\left(S_{1} \mid b=1\right) \operatorname{Pr}(b=1) \\
= & \frac{1}{2} \operatorname{erf~c}\left(\frac{\theta-\mu_{0}}{\sqrt{2 \sigma_{0}^{2}}}\right) \operatorname{Pr}\left(S_{0} \mid b=0\right)+\frac{1}{2} \operatorname{erf} \mathrm{c}\left(\frac{\mu_{1}-\theta}{\sqrt{2 \sigma_{1}^{2}}}\right) \\
& +\frac{1}{2}\left(1-\operatorname{erfc}\left(\frac{\mu_{1}-\theta}{\sqrt{2 \sigma_{1}^{2}}}\right)\right) \operatorname{Pr}\left(S_{1} \mid b=0\right)
\end{aligned}
$$

where $\operatorname{Pr}\left(S_{0} \mid b=0\right)=1-\operatorname{Pr}\left(S_{1} \mid b=0\right)$.

\section{NUMERICAL RESULTS}

Two classes of code weight $k_{1}$ and $k_{2}$ are considered in our numerical results. We assume that the code sizes of the codewords with weight $k_{1}$ and $k_{2}$ are $M_{1}$ and $M_{2}$, respectively. Table I lists the number of cardinalities of the two proposed $(n$, $\left.\left\{k_{1}, k_{2}\right\}\right)$-OOCs and conventional variable-weight $\left(n,\left\{k_{1}, k_{2}\right\}\right.$, $1,1,\{2 / 3,1 / 3\})$-OOCs [11], in which we assume the ratio of the code sizes is $M_{1}: M_{2}=2: 1$. Since the lower bound of code size of the first scheme does not depend on the ratio of 
TABLE II

SYSTEM PARAMETERS

\begin{tabular}{llll}
\hline \hline Name & Symbol & value & unit \\
\hline light wavelength & & 1.3 & $\mu m$ \\
APD quantum efficiency & $\eta$ & 0.6 & \\
APD gain & $G$ & 100 & \\
APD effective ionization ratio & $k_{e f f}$ & 0.02 & \\
APD bulk leakage current & $I_{b}$ & 0.1 & $\mathrm{nA}$ \\
APD surface leakage current & $I_{s}$ & 10 & $\mathrm{nA}$ \\
chip duration & $T_{c}$ & 0.1 & $\mathrm{~ns}$ \\
bit rate & $T_{b}=\frac{1}{n T c}$ & & \\
receiver noise temperature & $T_{r}$ & 300 & $K$ \\
receiver load resistor & $R_{L}$ & 1030 & $\Omega$ \\
\hline
\end{tabular}

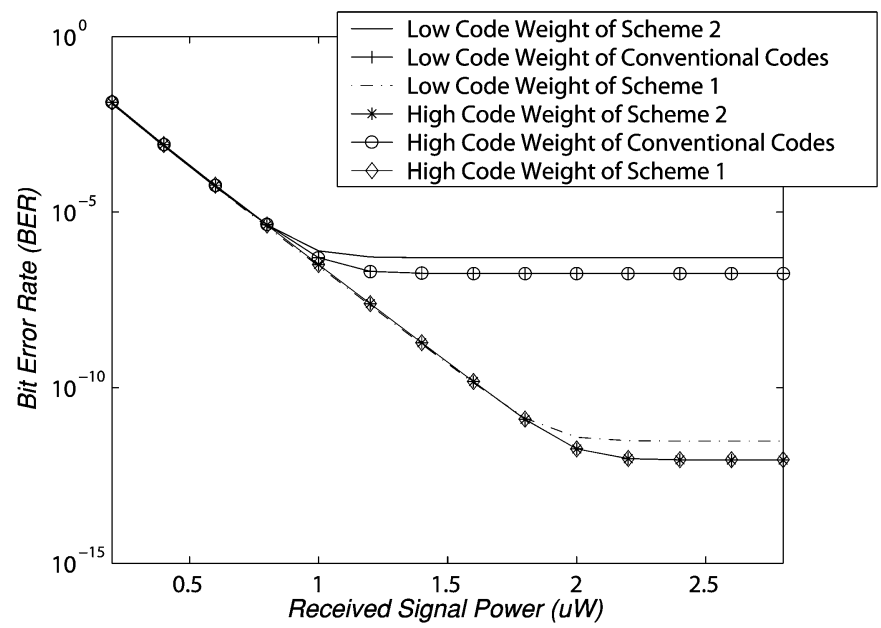

Fig. 2. BER of the variable-weight $(4161,\{9,5\})$-OOCs versus the received signal power when $\left\{m_{1}, m_{2}\right\}=\{40,20\}$.

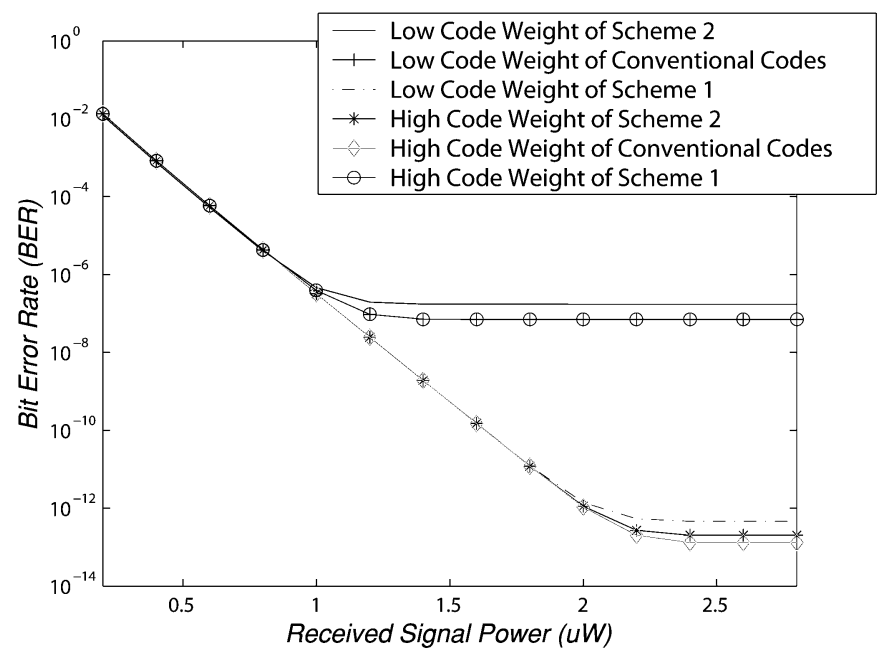

Fig. 3. BER of the variable-weight $(4161,\{9,5\})$-OOCs versus received signal power when $\left\{m_{1}, m_{2}\right\}=\{20,40\}$.

$M_{1}$ and $M_{2}$, we would not compare it with the upper bound of that from conventional codes. Moreover, the codes from the second scheme have larger code size than the conventional codes and the codes generated by the first scheme. This is because some of the cross correlation $\lambda_{c}$ of the codes generated by the second scheme is one, and some of them are relaxed to two.

The system parameters are listed in Table II. Figs. 2 and 3 show the BER of the variable-weight $(4161,\{9,5\})$-OOCs

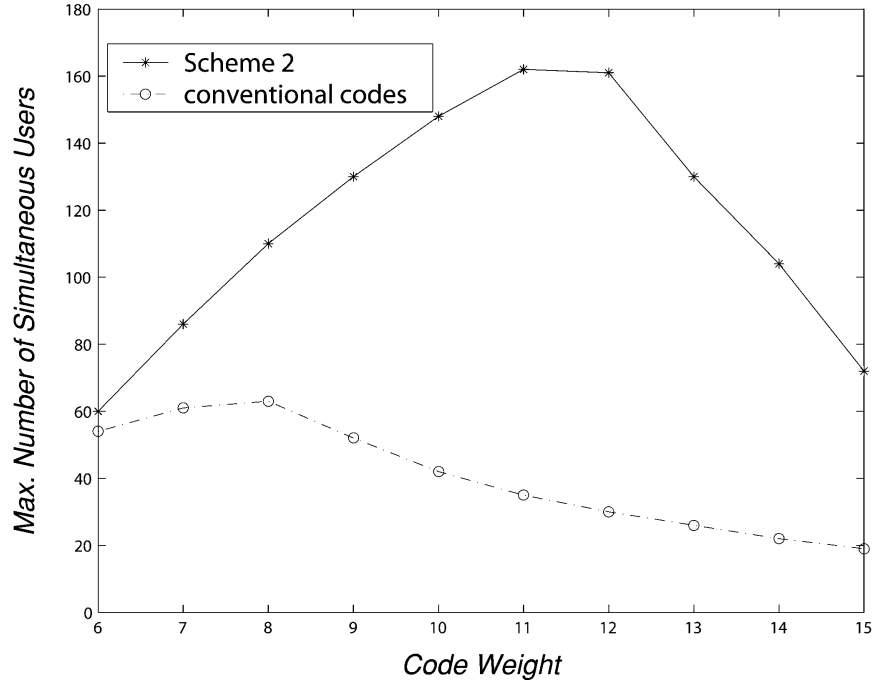

Fig. 4. Maximum number of high-weight users versus high code weight $k$ of the $(4161,\{k, 5\})$-OOCs when the received signal power $=2.4 \mu \mathrm{W}$ and $\mathrm{BER} \leq 10^{-9}$.

versus the received signal power when the number of simultaneous users are $\left\{m_{1}, m_{2}\right\}=\{40,20\}$ and $\{20,40\}$, respectively. We find that the BER performances of the codes generated by the first scheme and the conventional codes are identical because both of them have the same correlation constraint. The BER of the codes from the second scheme is slightly larger than that of the previous ones when the received signal power is large. However, the code size based on the second scheme is much larger than that of the conventional codes at the expense of little BER degradation. Fig. 4 illustrates the maximum number of high-weight users versus high code weight $k$ of the $(4161,\{k, 5\})$-OOCs when the number of low-weight users is 20 , received signal power $=2.4 \mu \mathrm{W}$, and BER $<10^{9}$. The result shows that the maximum number of high-weight users of the second scheme is much larger than that of the conventional codes. This is because the user number of conventional codes is limited by the code size. Therefore, both BER performance and the code size should be considered in the O-CDMA systems in order to have maximum capacity.

Next, we compare the performances of the codes from the second construction scheme and the conventional codes under the condition of about the same code size. We take the code length of the codewords equal to 4161 , which is the length of the Singer difference set with $q=2$ and $a=6$. Then, we can choose $\{9,8\}$ as the code weights of codes from the second construction scheme. The code size of the $(4161,\{9,8\})$-OOCs of the second scheme is 520 . For comparison, we take the conventional variable-weight $(4161,\{5,3\}, 1,1,\{1 / 6,5 / 6\})$-OOCs because the code size is about 500. Fig. 5 shows the performances of the conventional variable-weight $(4161,\{5,3\}, 1$, $1,\{1 / 6,5 / 6\})$-OOCs and the proposed $(4161,\{9,8\})$-OOCs when $\left\{m_{1}, m_{2}\right\}=\{50,100\}$. We observe that the BER performance of the proposed codes is much better than that of the conventional codes. Next, we investigate the effect of the number of simultaneous users on the system performance. We analyze the performance of the system using $(4161,\{9,8\})$-OOCs of the second construction scheme and 


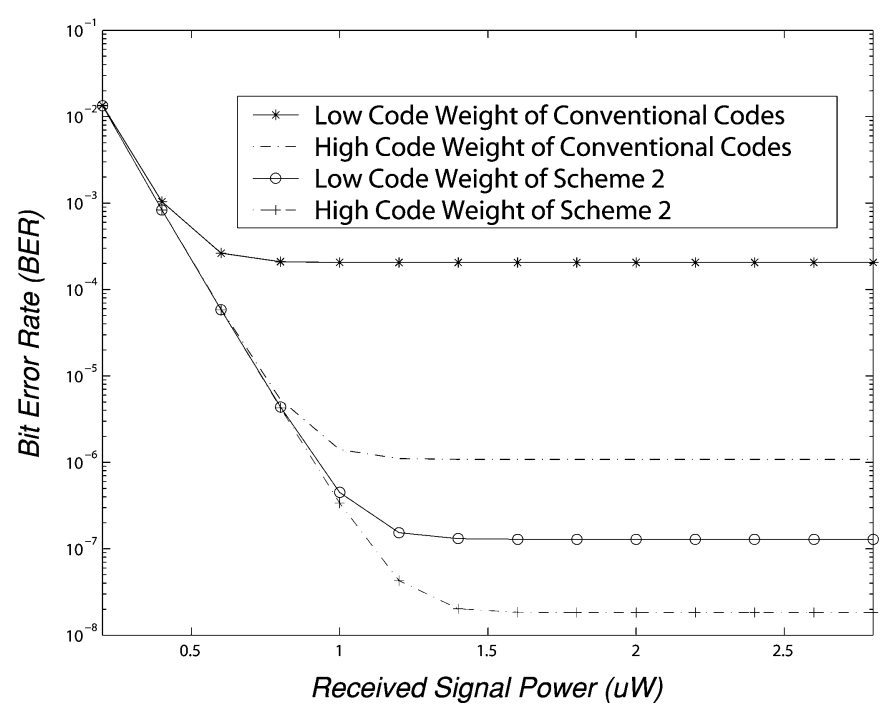

Fig. 5. BER of the codes from scheme 2 and conventional codes versus the received signal power when $\left\{m_{1}, m_{2}\right\}=\{50,100\}$.

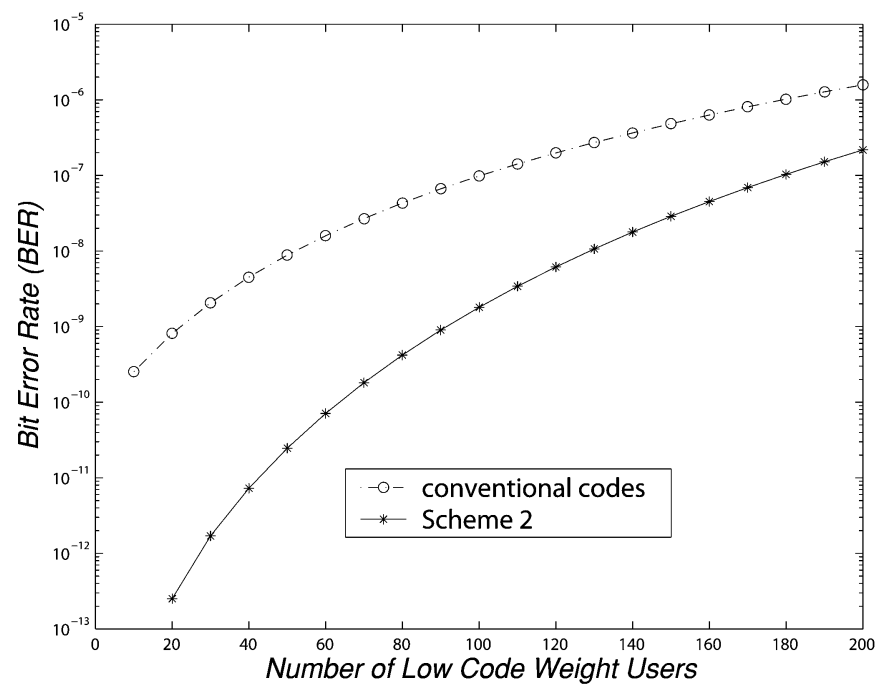

Fig. 6. BER performance of the high-code-weight user versus the number of simultaneous users with low code weight when the received signal power $=$ $2.4 \mu \mathrm{W}$.

that using conventional $(4161,\{5,3\}, 1,1,\{1 / 6,5 / 6\})$-OOCs when the number of high-code-weight users is 20 . Fig. 6 shows the BER performance of the high-code-weight user versus the number of simultaneous users with low code weight when the received signal power $=2.4 \mu \mathrm{W}$. At BER $\leq 10^{-9}$, the system with the conventional codes has 23 simultaneous low-code-weight users. On the other hand, the system with the proposed codes has 92 simultaneous low-code-weight users.

\section{CONCLUSION}

For the next-generation multimedia network, the QoS of the multiple Internet traffic must be guaranteed. Therefore, the variable-weight OOCs can be used as the spreading codes in the O-CDMA systems to meet the QoS requirements. On the other hand, the variable-weight OOCs can have larger code size than that of constant-weight OOCs. Two construction schemes of variable-weight OOCs are investigated in this paper. The first scheme uses PBD to produce a family of blocks with unequal block size. The second scheme uses packing design on constant-weight OOCs and partitions a constant-weight codeword into a family of codes with a smaller code weight. The code sizes of the two proposed codes are discussed in this paper. The BER performance of these two proposed codes are also evaluated analytically. Numerical results show that the codes from the first scheme and the conventional codes have identical performance because both of them have the same correlation constraint. The codes generated from the second scheme have much larger code size than that of the previous ones. The maximum number of users of the codes from the second scheme is larger than that of conventional codes. Meanwhile, the BER performance of the system using the codes of the second construction scheme is also better than that of the conventional codes under the condition of about the same code size.

\section{REFERENCES}

[1] G. Kramer and G. Pesavento, "Ethernet passive optical network (EPON): Building a next-generation optical access network," IEEE Commun. Mag., vol. 40, no. 2, pp. 66-73, Feb. 2002.

[2] B. G. Ahn and Y. Park, "A symmetric-structure CDMA-PON system and its implementation," IEEE Photon. Technol. Lett., vol. 14, no. 9, pp. 1381-1383, Sep. 2002.

[3] J. A. Salehi, "Code division multiple-access techniques in optical fiber networks-part I: Fundamental principles," IEEE Trans. Commun., vol. 37, no. 8, pp. 824-833, Aug. 1989.

[4] C. J. Colbourn and J. H. Dinitz, CRC Handbook of Combinatorial Designs. Boca Raton, FL: CRC, 1996.

[5] A. Pott, Finite Geometry and Character Theory. New York: Springer, 1995.

[6] J. Schönheim, "On maximal system of k-tuples," Studia Sci. Math. Hungar, vol. 1, pp. 363-368, 1966.

[7] J. A. Salehi and C. A. Brackett, "Code division multiple-access techniques in optical fiber networks-part II: systems performance analysis," IEEE Trans. Commun., vol. 37, no. 8, pp. 834-842, Aug. 1989.

[8] G. C. Yang and T. E. Fuja, "Optical orthogonal codes with unequal auto-and cross-correlation constrains," IEEE Trans. Inf. Theory, no. 1, pp. 96-106, Jan. 1995.

[9] C. S. Weng and J. Wu, "Optical orthogonal codes with nonideal cross correlation," J. Lightw. Technol., vol. 19, no. 12, pp. 1856-1863, Dec. 2001.

[10] - "Optical orthogonal codes with large cross-correlation and their performance bound for asynchronous optical CDMA systems," $J$. Lightw. Technol., vol. 21, no. 3, pp. 735-742, Mar. 2003.

[11] G. C. Yang, "Variable-weight optical orthogonal codes for CDMA networks with multiple performance requirements," IEEE Trans. Commun., vol. 44, no. 1, pp. 47-55, Jan. 1996.

[12] P. Erdös, R. C. Mullin, V. T. SóS, and D. R. Stinson, "Finite linear spaces and projective planes," Discrete Mathematics, vol. 47, pp. 49-62, 1983

[13] M. Buratii, "Pairwise balanced designs from finite fields," Discrete Mathematics, vol. 208, pp. 103-117, Oct. 1999.

[14] T. Beth, D. Jungnickel, and H. Lenz, Design Theory. Cambridge, U.K.: Cambridge Univ. Press, 1999.

[15] P. Erdös, J. C. Fowler, V. T. SóS, and R. M. Wilson, “On 2-designs," J. Combinatorial Theory, Series A, vol. 38, pp. 131-142, 1985.

[16] R. G. Stanton, "Minimal pairwise balanced designs," Discrete Mathematics, vol. 77, pp. 317-321, 1989.

[17] D. R. Stinson, "Applications and generalizations of the variance method in combinatorial designs," Utilitas Mathematica, vol. 22, pp. 323-333, 1982.

[18] T. Ohtsuki, "Performance analysis of direct-detection optical asynchronous CDMA systems with double optical hard-limiters," J. Lightw. Technol., vol. 15, no. 3, pp. 452-457, Mar. 1997.

[19] H. M. Kwon, "Optical orthogonal code-division multiple-access systempart I: APD noise and thermal noise," IEEE Trans. Commun., vol. 42, no. 7, pp. 2470-2479, Jul. 1994. 
[20] A. Stok and E. H. Sargent, "Lighting the local area: optical code-division multiple access and quality of service provisioning," IEEE Network, vol. 14, no. 6, pp. 42-46, Nov./Dec. 2000.

[21] F. E. Bennett, C. J. Colbourn, and R. C. Mullin, "Quintessential pairwise balanced designs," J. Statistical Planning Inference, vol. 72, pp. 15-66, 1998.

[22] A. C. H. Ling and C. J. Colbourn, "Pairwise balanced designs with block size 8, 9 and 10," J. Combinatorial Theory, Series A, vol. 77, pp. 228-245, 1997.

[23] M. Greig, "Designs from projective planes and PBD bases," J. Combinatorial Designs, vol. 7, pp. 341-374, 1999.

[24] —_, "Some pairwise balanced designs," Electronic J. Combinatorics, vol. 7 , no. 1, pp. 13-33, 2000.
[25] P. Erdös and H. Hanani, "On a limit the theorem in combinatorial analysis," Publications Mathematical Debrecen, vol. 10, pp. 10-13, 1963.

Fong-Ray Gu, photograph and biography not available at the time of publication.

Jingshown Wu (S'73-M'78-SM'99), photograph and biography not available at the time of publication. 